\title{
Longitudinal esophageal muscle dysfunction reported in eosinophilic esophagitis
}

Selective dysfunction of longitudinal esophageal muscles exists in patients with esophageal esophagitis (EE), according to a new study.

The pathology and physiology of EE is poorly defined. Korsapti and colleagues decided to study longitudinal esophageal muscles in EE after they noticed some surprising results when examining an apparently healthy individual.

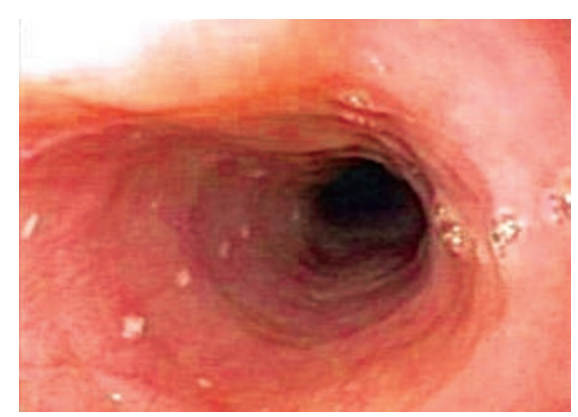

G. T. Furuta, The Children's Hospital Denver, Aurora, co
Longitudinal muscle function was abnormal in this patient and the team predicted the individual had EE; they were correct in their diagnosis.

The researchers combined ultrasound imaging with manometry to record longitudinal and circular muscle function simultaneously during swallow-induced peristalsis in patients with EE $(n=10)$, and healthy individuals $(n=10)$. Longitudinal muscle thickness (a marker of muscle contraction) is markedly reduced, and muscle response to edrophonium (a stimulant of muscle contraction) is blunted in patients with EE. Mild asynchrony of circular and longitudinal muscle contractions was also apparent in patients with EE, but not in healthy controls. "We believe our study is the first to show that there is selective dysfunction of the longitudinal muscle in EE" explains Ravinder Mittal, part of the research team, "in fact, EE is the first clinical disorder in which one muscle (circular muscle) functions relatively normally in the esophagus but the other (longitudinal muscle) shows severe dysfunction."

\section{4 ...certain products of} inflammation released in EE may affect longitudinal muscle function selectively 77

The team suspects that certain products of inflammation released in EE may affect longitudinal muscle function selectively.

Rachel Jones

Original article Korsapati, H. et al. Dysfunction of the longitudinal muscles of the oesophagus in eosinophilic esophagitis. Gut 58, 1056-1062 (2009). 